\title{
Cingulate Hypoactivity in Cocaine Users During a GO-NOGO Task as Revealed by Event-Related Functional Magnetic Resonance Imaging
}

\author{
Jacqueline N. Kaufman, ${ }^{1}$ Thomas J. Ross, ${ }^{1}$ Elliot A. Stein, ${ }^{1}$ and Hugh Garavan ${ }^{1,2}$ \\ ${ }^{1}$ Medical College of Wisconsin, Department of Psychiatry, Milwaukee, Wisconsin 53226, and ${ }^{2}$ Trinity College, Department of Psychology and Institute of \\ Neuroscience, Dublin 2, Ireland
}

\begin{abstract}
Although extensive evidence exists for the reinforcing properties of drugs of abuse such as cocaine, relatively less research has addressed the functional neuroanatomical correlates of the cognitive sequelae of these drugs. We present a functional magnetic resonance imaging study of a GO-NOGO task in which successful performance required prepotent behaviors to be inhibited. Significant cingulate, presupplementary motor and insula hypoactivity was observed for both successful NOGOs and errors of commission in chronic cocaine users relative to cocaine-naive controls. This attenuated response, in the presence of comparable activation levels in other task-related cortical areas, suggests cortical and psychological specificity in the locus of drug abuse-related cognitive dysfunction. The results suggest that addiction may be accompanied by a disruption of brain structures critical for the higher-order, cognitive control of behavior.
\end{abstract}

Key words: inhibitory control; cocaine; fMRI; anterior cingulate; GO-NOGO; addiction

\section{Introduction}

Cocaine has been identified as one of the most powerful reinforcers currently known (Kuhar et al., 1991). Similar to the reinforcing properties of many drugs of abuse, the pharmacological effects of cocaine have been linked to the modulation of dopamine release, particularly within terminal fields of the mesocorticolimbic dopaminergic pathways such as the nucleus accumbens (Koob and Bloom, 1988; Wise, 1996; Di Chiara, 1999). These pharmacological effects of abused drugs do not, however, directly explain the cognitive deficits often seen in chronic drug users. For example, chronic cocaine abusers display impairment on tests of memory function, attention, and inhibitory control (O'Malley et al., 1992; Easton and Bauer, 1997; Di Sclafani et al., 1998; Fillmore and Rush, 2002). Although a great deal of preclinical and clinical research has focused on the direct action of cocaine on the reward systems of the brain (Wise, 1996; Di Chiara, 1999), comparatively few studies have examined the functional neuroanatomical regions associated with these observed behavioral correlates of cocaine use. Consequently, an unresolved question concerns the role that cognitive dysfunction and its associated neuroanatomical changes might play in the maintenance of drug abuse.

Poor inhibitory control is a common symptom of a number of pathologies that fall under the umbrella of impulsivity disorders.

\footnotetext{
Received March 11, 2003; revised June 9, 2003; accepted June 12, 2003.

This work was supported by United States Public Health Service Grant DA14100 and General Clinic Research Center (GCRC) Grant M01 RR00058. We gratefully acknowledge the assistance of Stacy Claesges, Michael San Fillipo, the research nusring staff at GCRC of Froedtert Memorial Lutheran Hospital, and the comments of two anonymous reviewers.

Correspondence should be addressed to Dr. Hugh Garavan, Department of Psychology, Trinity College Dublin, Dublin 2, Ireland. E-mail: hugh.garavan@tcd.ie.

T. J. Ross's and E. A. Stein's present address: National Institute on Drug Abuse-Intermural Research Program, Neuroimaging Research Branch, 5500 Nathan Shock Drive, Baltimore, MD 21224.

Copyright $\odot 2003$ Society for Neuroscience $\quad$ 0270-6474/03/237839-05\$15.00/0
}

Pathological gambling, obsessive-compulsive disorder (OCD), attention deficit hyperactivity disorder, Tourrette syndrome, and a variety of traumatic brain injuries (Stewart and Tannock, 1999; Hollander and Rosen, 2000; Johannes et al., 2001; Ursu et al., 2001) share common symptoms of disinhibited behavior and loss of self-control. These cognitive constructs are also among the characteristics of drug addiction and suggest that executive dysfunction (i.e., impairment in the cognitive control of behavior) may be a core component of addiction (Lyvers, 2000). The failure to develop adequate inhibitory control and/or the loss ofpreviously developed inhibitory control can have a profound impact on the ability of an individual to gate prepotent, yet inappropriate and dangerous, behaviors such as using cocaine. Lesion studies and, more recently, brain imaging studies have implicated frontal and parietal cortex in inhibitory control (Konishi et al., 1999; Pliszka et al., 2000; Brass et al., 2001; Fuster, 2001; Rubia et al., 2001; Garavan et al., 2002). Whereas right prefrontal cortex (PFC) appears to be central to response inhibition (Garavan et al., 1999; Aron et al., 2003), the anterior cingulate and occipitotemporal regions have also frequently been reported (de Zubicaray et al., 2000; Braver et al., 2001; Liddle et al., 2001; Menon et al., 2001; Garavan et al., 2002; Watanabe et al., 2002).

The ability to inhibit inappropriate behaviors is complemented by action monitoring functions. For example, efficient executive control of behavior requires us to monitor our performance for errors or for high levels of task difficulty (e.g., conflict between competing responses) and then to adjust our behavior accordingly (Rabbitt, 1966; Botvinick et al., 2001). Thus, action monitoring helps ensure smooth and safe control of behavior. These monitoring functions have been anatomically dissociated from inhibitory control functions (Menon et al., 2001; Garavan et al., 2002) and have been localized to medial wall structures such as the anterior cingulate 
cortex (ACC) and pre-supplementary motor area (SMA) (Carter et al., 1998; Ullsperger and von Cramon, 2001).

A growing imaging literature suggests ACC and medial prefrontal dysregulation in chronic cocaine users as evidenced by anatomical changes as well as metabolic dysfunctions. For example, polysubstance users have been shown to have bilateral decreases in prefrontal gray matter (Liu et al., 1998). When compared with non-using control subjects, cocaine users also show gray matter decreases in ACC, insula, and superior temporal regions (Franklin et al., 2002). In addition to these anatomical differences, functional metabolic variations have been observed in cocaine users with decreased metabolic activity in the cingulate and orbitofrontal cortices (Volkow et al., 1993). In drug-abusing populations, as in other clinical populations, it is typically not possible to determine whether brain changes of these sorts predate the observed pathology or whether they are the result of the disordered behavior. Notwithstanding this common limitation, it is valuable to identify the neuroanatomical correlates of cognitive dysfunction to inform optimal treatment.

The present study attempted to probe the neuroanatomical functioning of these cognitive processes in cocaine users. GONOGO tasks in which the NOGO/GO ratio is low, thereby creating a response prepotency that is difficult to inhibit on NOGO trials, provide a useful test bed for assessing cortical activation for inhibitory control and action monitoring. Cocaine-using subjects have been shown to have impaired behavioral performance on GO-NOGO tasks (Fillmore and Rush, 2002; Fillmore et al., 2002); the functional imaging correlates of these behavioral findings would provide important information regarding the localization of these impairments. Consequently, both cocaine-naive control subjects and chronic cocaine-using subjects underwent functional magnetic resonance imaging (fMRI) scanning while performing a visually presented GO-NOGO task.

\section{Materials and Methods}

Participants. Of the 27 subjects who participated in this study, 13 were otherwise healthy, active cocaine users (five female; mean age of $37 \pm 4.5$ years; range of 27-44 years), and 14 were healthy nondrug users (10 female; mean age, $30 \pm 8.7$ years; range, $19-45$ years). Among cocaine users, the average number of years of cocaine use was 11.2 (range of 1-22 years). All participants were right-handed and reported no history of neurological symptoms. Subjects were fully informed of the nature of the research and provided written consent for their involvement in this study in accordance with the Institutional Review Board of the Medical College of Wisconsin. Urine samples were collected from all participants to test for pregnancy and drug use. All nondrug users had negative tests for all drugs, whereas all cocaine subjects returned positive screens for cocaine or its metabolites, indicating that they had used cocaine within the previous $72 \mathrm{hr}$. Ten of the 13 users were able to estimate their last use, which ranged from 18 to $72 \mathrm{hr}$ before the scan session, and no user displayed any overt behavioral signs of cocaine intoxication.

Stimuli. Stimuli consisted of a $1 \mathrm{~Hz}$ serial stream of alternating Xs and Ys. The stimuli were 5.08 and $6.35 \mathrm{~cm}$ in height for $\mathrm{X}$ and $\mathrm{Y}$, respectively. Subjects were instructed to press a button for each target stimulus while the stimulus was still present on the screen. NOGO stimuli, in which the target stimuli did not alternate (i.e., the second of two identical, successively presented target stimuli), required inhibition of the response. Subjects recommenced responding to alternating stimuli after the NOGO stimulus. There were $1180 \mathrm{GO}$ and 80 randomly distributed NOGO stimuli presented over four runs (Fig. 1).

Training. Before functional image acquisition, subjects were trained on the task in a quiet room. Training included four difficulty levels of the task, accomplished by varying the on-screen duration of the stimuli at $900,800,700$, or 600 msec: shorter stimulus durations, when combined with the instruction to respond while the stimulus was on-screen, yield

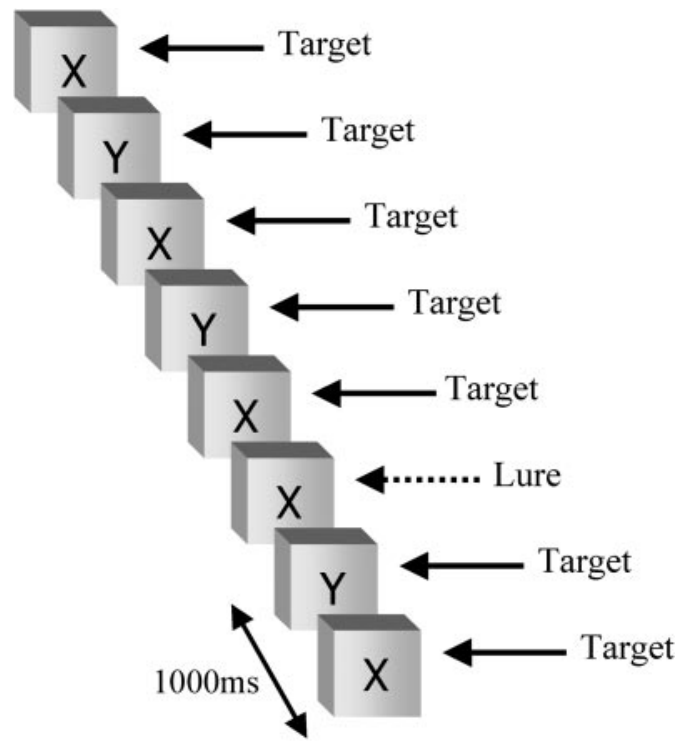

Figure 1. Subjects were presented with $1 \mathrm{~Hz}$ serial stream of alternating $X_{s}$ and $Y_{s}$ (GO stimuli), all of which were responded to with a button press. Periodic lure presentations (NOGO stimuli) in which the target did not alternate required an inhibition of a response.

increased numbers of commission errors (Garavan et al., 2002). A blank screen was displayed during the remainder of the $1 \mathrm{sec}$ stimulus display time to maintain the stimuli rate at $1 \mathrm{~Hz}$. Training durations were $\sim 30$ min but varied by subject because instruction pages were self-paced and the durations of rest periods during training were at the subject's discretion. For the imaging runs, task difficulty was tailored for each subject by selecting one of the four presentation rates to achieve comparable behavioral performance in drug users and nondrug users and to ensure nearequal numbers of successful inhibitions (STOPS) and commission errors (ERRORS). This tailoring was necessary to ensure that sufficient numbers of STOPS and ERRORS were available for the event-related analyses. Given that poorer inhibitory control performance would be expected in cocaine users, the tailoring sought to ensure that each group performed at similar levels of competence, thereby minimizing fatigue or frustration confounds. Subject placement in conditions based on training performance was as follows: the $600 \mathrm{msec}$ condition was run on $57 \%$ of the control subjects and $23 \%$ of the cocaine subjects; the $700 \mathrm{msec}$ condition was run on $14 \%$ of the control subjects and $38 \%$ of the cocaine subjects; the 800 msec condition was run on $14 \%$ of the control subjects and $8 \%$ of the cocaine subjects; and the 900 msec condition was run on $14 \%$ of the control subjects and $31 \%$ of the cocaine subjects.

$f M R I$ procedures. Participants were fitted with prism glasses that permitted them to view stimuli backprojected onto a screen at the end of the scanner bed. To minimize head movement and artifact in the coil during data acquisition, subject's heads were cushioned with padding. Wholebrain fMRI imaging was performed using a 1.5 T GE Signa scanner (GE Medical Systems, Waukesha, WI) equipped with a $30.5 \mathrm{~cm}$ internal diameter three-axis local gradient coil with insertable radiofrequency coils with transmit-receive capabilities. Contiguous $7 \mathrm{~mm}$ sagittal slices spanning the entire brain were collected using a gradient-echo, echo-planar pulse sequence (repeat time, $2000 \mathrm{msec}$; echo time, $40 \mathrm{msec}$; field of view, $240 \mathrm{~mm}$; $64 \times 64$ matrix; $3.75 \times 3.75 \mathrm{~mm}$ in-plane resolution). Highresolution spoiled gradient recalled acquisition at steady state (SPGR) anatomical images were collected before the functional data acquisition and were used for spatial normalization and subsequent activation localization.

fMRI data analysis. Imaging data were analyzed using the AFNI software package (Cox, 1996). Functional data were three-dimensional volume registered and corrected for differences in slice time acquisition. A deconvolution analysis calculated the hemodynamic response functions for STOPS and ERRORS that were modeled using a gamma-variate function. The modeling, using nonlinear regression, identified the best fitting 


\begin{tabular}{|c|c|c|c|c|c|c|}
\hline \multirow[b]{2}{*}{ Structure } & \multirow[b]{2}{*}{ Brodmann area } & \multirow[b]{2}{*}{ Hemisphere } & \multirow[b]{2}{*}{ Volume $(\mu l)$} & \multicolumn{3}{|c|}{ Center of mass } \\
\hline & & & & $x$ & $y$ & $z$ \\
\hline \multicolumn{7}{|l|}{ Successful inhibitions (STOPS) } \\
\hline \multicolumn{7}{|l|}{ Frontal lobe } \\
\hline \multirow[t]{2}{*}{ Middle frontal gyrus } & 9 & $\mathrm{R}$ & 667 & 44 & -21 & 28 \\
\hline & 6 & $\mathrm{R}$ & 259 & 35 & 1 & 50 \\
\hline Inferior frontal gyrus & 9 & $\mathrm{R}$ & 539 & 51 & -5 & 20 \\
\hline Superior frontal gyrus & 6 & $\mathrm{R}$ & 2739 & 3 & -5 & 52 \\
\hline Precentral gyrus & 6 & $\mathrm{R}$ & 336 & 39 & 4 & 42 \\
\hline \multirow[t]{3}{*}{ Cingulate gyrus } & 24 & $\mathrm{~L}$ & 359 & -6 & -26 & 22 \\
\hline & $24 / 32$ & $\mathrm{R}$ & 123 & 12 & -3 & 44 \\
\hline & $6 / 32^{*}$ & $\mathrm{R}$ & 663 & 9 & -28 & 32 \\
\hline \multicolumn{7}{|l|}{ Parietal lobe } \\
\hline \multirow[t]{2}{*}{ Inferior parietal lobule } & $7 / 40$ & $\mathrm{R}$ & 132 & 33 & 56 & 40 \\
\hline & 40 & $\mathrm{R}$ & 1018 & 49 & 38 & 42 \\
\hline \multicolumn{7}{|l|}{ Temporal lobe } \\
\hline Middle temporal gyrus & 21 & $\mathrm{R}$ & 141 & 57 & 44 & -2 \\
\hline \multicolumn{7}{|l|}{ Subcortical } \\
\hline \multirow[t]{2}{*}{ Putamen } & & $\mathrm{L}$ & 3587 & -23 & -2 & 6 \\
\hline & & $\mathrm{R}$ & 3277 & 26 & -9 & 4 \\
\hline Thalamus & & $\mathrm{R}$ & 448 & 7 & 14 & 5 \\
\hline Insula & $47^{* *}$ & $\mathrm{R}$ & 149 & 47 & -12 & -4 \\
\hline \multicolumn{7}{|l|}{ Failed inhibitions (ERRORS) } \\
\hline \multicolumn{7}{|l|}{ Frontal lobe } \\
\hline \multirow[t]{2}{*}{ Middle frontal gyrus } & 6 & $\mathrm{R}$ & 644 & 19 & 2 & 60 \\
\hline & 6 & $\mathrm{R}$ & 125 & 24 & -8 & 50 \\
\hline Medial frontal gyrus & $6^{* *}$ & $\mathrm{R}$ & 386 & 5 & -7 & 60 \\
\hline Superior frontal gyrus & 6 & $\mathrm{~L}$ & 108 & -4 & 9 & 56 \\
\hline Precentral gyrus & 6 & $\mathrm{R}$ & 150 & 42 & -6 & 39 \\
\hline Inferior frontal gyrus & $6^{* *}$ & $\mathrm{~L}$ & 522 & -45 & 2 & 31 \\
\hline Cingulate gyrus & $24^{* *}$ & $\mathrm{R}$ & 289 & 4 & 14 & 31 \\
\hline $\begin{array}{l}\text { Cingulate/medial frontal gyrus/ } \\
\text { superior frontal gyrus }\end{array}$ & $6 / 8 / 32^{*}$ & $\mathrm{R}$ & 3650 & 4 & 12 & 46 \\
\hline \multicolumn{7}{|l|}{ Parietal lobe } \\
\hline \multirow[t]{2}{*}{ Inferior parietal lobule } & 40 & $\mathrm{R}$ & 280 & 51 & -39 & 40 \\
\hline & 40 & $\mathrm{R}$ & 186 & 43 & -48 & 39 \\
\hline \multicolumn{7}{|l|}{ Temporal lobe } \\
\hline Middle temporal & $39 / 40$ & $\mathrm{R}$ & 108 & 47 & -45 & 27 \\
\hline Gyrus & 37 & $\mathrm{~L}$ & 130 & -50 & -57 & 5 \\
\hline \multicolumn{7}{|l|}{ Subcortical } \\
\hline Putamen & & $\mathrm{R}$ & 3194 & 25 & 8 & 4 \\
\hline Thalamus & & $\mathrm{L}$ & 117 & -11 & -18 & 11 \\
\hline Insula & $13^{* *}$ & $\mathrm{~L}$ & 1080 & -30 & 13 & 6 \\
\hline
\end{tabular}

Positive center-of-mass coordinates for $x, y$, and $z$ refer to locations right $(x)$, posterior $(y)$, and superior $(z)$ to the anterior commissure. Brain regions in which between-group comparisons revealed significant differences in activation between cocaine users and controls are denoted: ${ }^{*} p \leq 0.05 ;{ }^{* *} p \leq 0.01$. R, Right; L, left.

gamma-variate function for each voxel rather than constraining all hemodynamic responses to a standard, prespecified shape. The area under the curve of this hemodynamic model was calculated for each voxel and expressed as a percentage of the area under the baseline (representing tonic task-related processes). Functional maps were transformed into stereotaxic space based on the atlas of Talairach and Tournoux (1988) and spatially blurred with a $4.2 \mathrm{~mm}$ full-width half-maximal isotropic Gaussian kernel. Activation maps were created for both STOPS and ERRORS for each group based on one-sample $t$ tests against the null hypothesis of no activation changes. Data simulations, created by randomly selecting STOP and ERROR locations within the time series and exactly repeating all subsequent analyses, identified the voxelwise threshold at which there was a $5 \%$ chance of a false positive cluster of activation $(t=$ 4.95 with a minimal cluster size of $100 \mu \mathrm{l}$ ). The activation maps of the users and control subjects were combined by condition as OR (either/or data) maps, and between group comparisons were performed on the mean activations of these clusters.

\section{Results}

\section{Performance analysis}

Prescanning performance results revealed poorer inhibitory control in the cocaine users (51 vs 39 commission errors; $t_{(25)}=2.3 ; p \leq$
0.03). Despite efforts to tailor task difficulty individually during scanning, cocaine users made significantly more commission errors $\left(48\right.$ vs $\left.36 ; t_{(25)}=2.79 ; p<0.01\right)$ and omission errors $\left(58\right.$ vs $3 ; t_{(25)}=$ $4.13 ; p<0.0004)$ than noncocaine users. However, the event-related design of the fMRI experiment allowed us to compare the two groups separately on successful behavioral inhibitions (STOPS) and failed inhibitions (ERRORS), thereby removing performance confounds from the brain activation maps.

\section{Functional analysis}

Activated areas were primarily in the right hemisphere and included dorsolateral PFC, ACC, inferior parietal lobule, and bilateral putamen (Table 1). The activation patterns of cocaine users were similar to controls, but, in some cases, the volume of activation was smaller in users (Fig. 2). The fMRI data revealed significantly less mean activation in cocaine users for STOPS in the anterior cingulate and right insula when compared with noncocaine users (Fig. 3). Notably, other areas, including those suggested previously to be important for inhibitory control (e.g., right inferior parietal lobule and right dorsolateral prefrontal cor- 
tex) (Garavan et al., 1999, 2002; Konishi et al., 1999; Fuster, 2001; Rubia et al., 2001), did not differ in activity between the groups for STOPS, underscoring the specificity of the hypoactive areas. In addition, a number of anterior cingulate clusters, as well as the right medial frontal gyrus/pre-SMA, left insula, and left inferior frontal gyrus were also significantly hypoactive in users relative to nonusers for ERRORS (Fig. 3) (for a complete list of structures activated by this task, see Table 1).

The levels of difficulty performed by the two groups were not equivalent. To determine whether this may underlie the observed effects, an analysis between matched groups (i.e., the subset of the present sample that can be chosen to ensure equal representation of each level of task difficulty) was performed, and similar patterns of hypoactivity were observed in the cocaine users. Additionally, multivariate ANOVA was run with all subjects examining the mean activation for each region of interest used in the analyses for both successful inhibitions and errors. Although no group $\times$ rate effect was seen, a group effect was present for both incorrect and correct (as expected per the overall findings of this study). There was also no effect for rate. This would suggest that there is not an interaction based on rate of presentation and that group differences persist even when rate is factored in.

\section{Discussion}

These data demonstrate that certain cortical areas, especially midline areas of the anterior cingulate that are critical for cognitive control, are less responsive in chronic cocaine users. STOP-related hypoactivity was observed in the right insula and a rostral region of the ACC, two regions that have been identified with emotional processes (Whalen et al., 1998). The ACC has also been implicated previously in inhibitory control (Casey et al., 1996; Ponesse et al., 1998). It has been suggested to play a critical role in urgent inhibitions (i.e., when time pressures preclude the involvement of a "controlled" response inhibition mediated by dorsolateral prefrontal areas) and has been shown to be relied on more by highly absentminded subjects (Garavan et al., 2002). Furthermore, we demonstrated that ACC function is compromised not only for successful STOPS but also for ERRORS. Others (Carter et al., 1998; Kiehl et al., 2000) have observed error-related activations in both the ACC and left prefrontal cortex, two areas observed to be hypoactive in cocaine users. This internal replication of ACC hypoactivity in cocaine users in the presence of comparable activation levels in many other taskrelated cortical areas demonstrates that differences in cortical function in users are anatomically specific and not ubiquitous. These results, therefore, enable us to link existing evidence of cognitive control impairment in addicts with the existing evidence of ACC dysfunction in this group.

Alterations in blood oxygenation level-dependent response in these midline action-monitoring regions have been demonstrated in other pathological populations. For example, the ACC is hyperactive in patients suffering from OCD and is hypoactive in schizophrenic patients (Carter et al., 1998; Ursu et al., 2001). These findings are consistent with the clinical profiles of these groups, suggesting an overactive action monitoring system and a heightened attentiveness to corrective behavior in OCD, and a failure to properly monitor and integrate stimuli in the environment in schizophrenia. Similarly, the hypoactivity of cocaine users would appear consistent with their pathological drug use pattern. Reduced inhibitory control, diminished action monitoring, and diminished responsivity to one's errors may represent an executive function profile of cocaine users that may, at its least, serve to prolong the maintenance of drug abuse. Recently, similar findings of ACC hypoactivity have been observed in opiate addicts (S. D. Forman, personal communication).

These dysexecutive sequelae of drug abuse suggest that cocaine users may be compromised in the endogenous and volitional control of their behavior. Consequently, their behavior may be disproportionately determined by environmental contingencies, environmental cues (e.g., drug craving cues), and au- 
tomatized or habitual behaviors. The effect of this would be to compound the maintenance of drug abuse: if chronic cocaine users are especially influenced by environmental contingencies and cues, then an inhibitory dysfunction may reduce their capacity to inhibit these external influences. Although these findings are quite consistent with previous reports of metabolic and neuroanatomical dysfunctions in cocaine users, the current design does not permit an examination of the time course of these changes. In effect, it is not possible to determine whether these changes predate the overt expression of a cocaine addiction or whether they are the outcome of a history of cocaine abuse. Additionally, although the current study has demonstrated a significant ACC dysfunction relating to one aspect of inhibitory control, namely the response inhibitions required of a GO-NOGO task, it remains to be seen whether other types of inhibitory control (Bechara et al., 2001; Bechara, 2003) would show a similar deficit. These caveats notwithstanding, an emerging knowledge of the cognitive profile of cocaine users and its associated functional neuroanatomy may inform optimal therapeutic interventions and help identify casual users most at risk for becoming drug dependent.

\section{References}

Aron AR, Fletcher PC, Bullmore ET, Sahakian BJ, Robbins TW (2003) Stopsignal inhibition disrupted by damage to right inferior frontal gyrus in humans. Nat Neurosci 6:115-116.

Bechara A (2003) Risky business: emotion, decision-making, and addiction. J Gambl Stud 19:23-51.

Bechara A, Dolan S, Denburg N, Hindes A, Anderson SW, Nathan PE (2001) Decision-making deficits, linked to a dysfunctional ventromedial prefrontal cortex, revealed in alcohol and stimulant abusers. Neuropsychologia 39:376-389.

Botvinick MM, Braver TS, Barch DM, Carter CS, Cohen JD (2001) Conflict monitoring and cognitive control. Psychol Rev 108:624-652.

Brass M, Zysset S, von Cramon DY (2001) The inhibition of imitative response tendencies. NeuroImage 14:1416-1423.

Braver TS, Barch DM, Gray JR, Molfese DL, Snyder A (2001) Anterior cingulate cortex and response conflict: effects of frequency, inhibition and errors. Cereb Cortex 11:825-836.

Carter CS, Braver TS, Barch DM, Botvinick MM, Noll D, Cohen JD (1998) Anterior cingulate cortex, error detection, and the online monitoring of performance. Science 280:747-749.

Casey BJ, Trainor R, Orendi J, Schubert A (1996) A functional Magnetic resonance imaging (fMRI) study of ventral prefrontal cortex mediation of response inhibition. Soc Neurosci Abstr 22:1107.

Cox RW (1996) AFNI: software for analysis and visualization of functional magnetic resonance neuroimages. Comput Biomed Res 29:162-173.

de Zubicaray GI, Andrew C, Zelaya FO, Williams SCR, Dumanoir C (2000) Motor response suppression and the prepotent tendency to respond: a parametric fMRI study. Neuropsychologia 38:1280-1291.

Di Chiara G (1999) Drug addiction as dopamine-dependent associative learning disorder. Eur J Pharmacol 375:13-30.

Di Sclafani V, Truran DL, Bloomer C, Tolou-Shams M, Clark HW, Norman D, Hannauer D, Fein G (1998) Abstinent chronic crack-cocaine and crack-cocaine/alcohol abusers evidence normal hippocampal volumes on MRI despite persistent cognitive impairments. Addict Biol 3:261-270.

Easton C, Bauer LO (1997) Neuropsychological differences between alcohol-dependent and cocaine-dependent patients with or without problematic drinking. Psychiatry Res 71:97-103.

Fillmore MT, Rush CR (2002) Impaired inhibitory control of behavior in chronic cocaine users. Drug Alcohol Depend 66:265-273.

Fillmore MT, Rush CR, Hays L (2002) Acute effects of oral cocaine on inhibitory control of behavior in humans. Drug Alcohol Depend 67:157-167.

Franklin TR, Acton PD, Maldjian JA, Gray JD, Croft JR, Dackis CA, O’Brian CP, Childress AR (2002) Decreased gray matter concentration in the insular, orbitofrontal, cingulate, and temporal cortices of cocaine patients. Soc Biol Psychiatry 51:134-142.
Fuster JM (2001) The prefrontal cortex - an update: time is of the essence Neuron 30:319-333.

Garavan H, Ross TJ, Stein EA (1999) Right hemispheric dominance of inhibitory control: an event-related functional MRI study. Proc Natl Acad Sci USA 96:8301-8306

Garavan H, Ross TJ, Murphy K, Roche RAP, Stein EA (2002) Dissociable executive functions in the dynamic control of behavior: inhibition, error detection and correction. NeuroImage 17:1820-1829.

Hollander E, Rosen J (2000) Impulsivity. J Psychopharmacol 14:S39-S44.

Johannes S, Wieringa BM, Mantey M, Nager W, Rada D, Muller-Vahl KR, Emrich HM, Dengler R, Munte TF, Dietrich D (2001) Altered inhibition of motor responses in Tourette Syndrome and Obsessive-Compulsive Disorder. Acta Neurol Scand 104:36-43.

Kiehl KA, Liddle PF, Hopfinger JB (2000) Error processing and the rostral anterior cingulate: an event-related fMRI study. Psychophysiology 37:216-223.

Konishi S, Nakajima K, Uchida I, Kikyo H, Kameyama M, Miyashita Y (1999) Common inhibitory mechanism in human inferior prefrontal cortex revealed by event-related functional MRI. Brain 122:981-991.

Koob GF, Bloom FE (1988) Cellular and molecular mechanisms of drug dependence. Science 242:715-723.

Liddle PF, Kiehl KA, Smith AM (2001) Event-Related fMRI study of response inhibition. Hum Brain Mapp 12:100-109.

Liu X, Matochik JA, Cadet JL, London ED (1998) Smaller volume of prefrontal lobe in polysubstance abusers: a magnetic resonance imaging study. Neuropsychopharmacology 18:243-252.

Lyvers M (2000) "Loss of control" in alcoholism and drug addiction: a neuroscientific interpretation. Exp Clin Psychopharmacol 8:225-249.

Menon V, Adleman NE, White CD, Glover GH, Reiss AL (2001) Errorrelated brain activation during a $\mathrm{Go} / \mathrm{NoG}$ o response inhibition task. Hum Brain Mapp 12:131-143.

O’Malley S, Adamse M, Heaton RK, Gawin FH (1992) Neuropsychological impairment in chronic cocaine abusers. Am J Drug Alcohol Abuse 18:131-144.

Pliszka SR, Liotti M, Woldorff MG (2000) Inhibitory control in children with attention deficit/hyperactivity disorder: event-related potentials identify the processing component and timing of an impaired rightfrontal response-inhibition mechanism. Biol Psychiatry 48:238-246.

Ponesse JS, Logan WJ, Schacher RS, Tannock R, Crawley AP, Mikulis DJ (1998) Functional neuroimaging of the inhibition of a motor response. NeuroImage 7:S972.

Rabbitt PM (1966) Errors and error correction in choice-response tasks. J Exp Psychol 71:264-272.

Rubia K, Russell T, Overmeyer S, Brammer MJ, Bullmore ET, Sharma T, Simmons A, Williams SC, Giampietro V, Andrew CM, Taylor E (2001) Mapping motor inhibition: conjunctive brain activations across different versions of go/no-go and stop tasks. NeuroImage 13:250-261.

Stewart JA, Tannock R (1999) Inhibitory control differences following mild head injury. Brain Cogn 41:411-416.

Talairach J, Tournoux P (1988) Co-planar stereotaxic atlas of the human brain. New York: Thieme Medical.

Ullsperger M, von Cramon DY (2001) Subprocesses of performance monitoring: a dissociation of error processing and response competition revealed by event-related fMRI and ERPs. NeuroImage 14:1387-1401.

Ursu S, van Veen V, Siegle G, MacDonald A, Stenger A, Carter C (2001) Executive control and self-evaluation in obsessive-compulsive disorder: an event related fMRI study. Paper presented at the Cognitive Neuroscience Society Meeting, New York, NY, March.

Volkow ND, Fowler JS, Wang GJ, Hitzemann R, Logan J, Schlyer DJ, Dewey SL, Wolf AP (1993) Decreased dopamine D2 receptor availability is associated with reduced frontal metabolism in cocaine abusers. Synapse 14:169-177.

Watanabe J, Sugiura M, Sato K, Sato Y, Maeda Y, Matsue Y, Fukuda H, Kawashima R (2002) The human prefrontal and parietal association cortices are involved in NO-GO performances: an event-related fMRI study. NeuroImage 17:1207-1216.

Whalen PJ, Bush G, McNally RJ, Wilhelm S, McInerney SC, Jenike MA, Rauch SL (1998) The emotional counting Stroop paradigm: a functional magnetic resonance imaging probe of the anterior cingulate affective division. Biol Psychiatry 44:1219-1228.

Wise RA (1996) Addictive drugs and brain stimulation reward. Annu Rev Neurosci 19:319-340. 